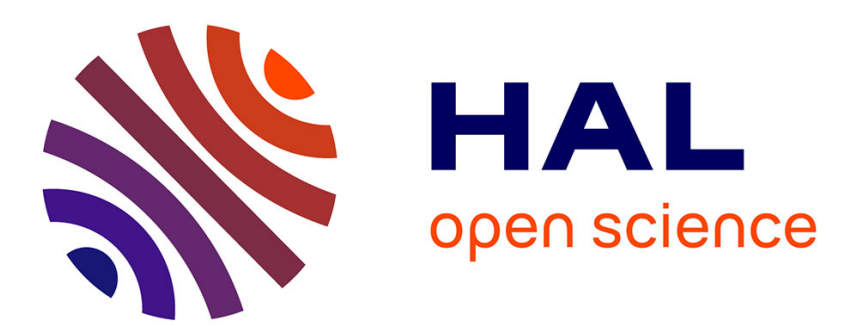

\title{
Laser Induced Fluorescence Observation of the Ion Velocity Distribution Function Modifications Induced by Electrostatic Ion Shock Waves
}

\author{
G. Bachet, L. Chérigier, C. Arnas-Capeau, F. Doveil, R. Stern
}

\section{- To cite this version:}

G. Bachet, L. Chérigier, C. Arnas-Capeau, F. Doveil, R. Stern. Laser Induced Fluorescence Observation of the Ion Velocity Distribution Function Modifications Induced by Electrostatic Ion Shock Waves. Journal de Physique III, 1996, 6 (9), pp.1157-1165. 10.1051/jp3:1996175 . jpa-00249514

HAL Id: jpa-00249514

https://hal.science/jpa-00249514

Submitted on 1 Jan 1996

HAL is a multi-disciplinary open access archive for the deposit and dissemination of scientific research documents, whether they are published or not. The documents may come from teaching and research institutions in France or abroad, or from public or private research centers.
L'archive ouverte pluridisciplinaire HAL, est destinée au dépôt et à la diffusion de documents scientifiques de niveau recherche, publiés ou non, émanant des établissements d'enseignement et de recherche français ou étrangers, des laboratoires publics ou privés. 


\title{
Laser Induced Fluorescence Observation of the Ion Velocity Distribution Function Modifications Induced by Electrostatic Ion Shock Waves
}

\author{
G. Bachet, L. Chérigier, C. Arnas-Capeau, F. Doveil $\left({ }^{*}\right)$ and R.A. Stern $\left({ }^{* *}\right)$ \\ Équipe Turbulence Plasma $\left({ }^{* * *}\right)$, Université de Provence, Institut Méditerranéen de Technologie, \\ 13451 Marseille Cedex 20, France
}

(Received 22 February 1996, revised 11 June 1996, accepted 13 June 1996)

PACS.52.35.Tc - Shock waves
PACS.52.70.Kz - Optical (ultraviolet, visible, infrared) measurements
PACS.52.50.Dg - Plasma sources

\begin{abstract}
Time resolution is added to a Laser Induced Fluorescence (LIF) diagnostic used to probe plasma ions. It is applied to investigate the effects induced by the propagation of electrostatic ion shock waves in a low density collisionless argon plasma. Preliminary results show the first LIF observation of the velocity distribution function of the "precursor" ions reflected by the shock front.
\end{abstract}

\section{Introduction}

Collisionless ion shock waves, associated with the non-linear saturation of electrostatic ion acoustic waves propagating in a low density plasma, have been extensively studied [1-8].

Between the plasma laser diagnostics [9], the Laser Induced Fluorescence (LIF) diagnostic $[10,11]$ is a means to non-intrusively probe plasmas and to measure the ion velocity distribution function with unprecedented resolutions in velocity (a few $\mathrm{m} \mathrm{s}^{-1}$ ) and space (a few $\mathrm{mm}^{3}$ ). This diagnostic based on the Doppler effect uses the fact that an ion in a long lived time state (metastable, or better fundamental) can be excited by a laser to a radiative state which decays by emitting fluorescence light. Usually this diagnostic gives the time averaged distribution function.

To experimentally study relaxation processes or instabilities, it is necessary to add time resolution. Due to the poor signal to noise ratio of the fluorescence, the observed phenomenon must be repetitive; the time resolution is then obtained by using, after the fluorescence light detector, a boxcar or a multichannel analyzer synchronized with the observed phenomenon. With this time resolution, the LIF diagnostic offers a powerful tool to probe the ion plasma feature. It can, in particular, be applied to study the turbulence or the instabilities associated with the propagation of collisionless electrostatic ion shock waves and to test theoretical models.

$\left(^{*}\right)$ Author for correspondence

$\left({ }^{* *}\right)$ Permanent address: Department of Physics, University of Colorado, Boulder, CO 80309, U.S.A.

$\left({ }^{* * *}\right)$ URA 773 CNRS

(C) Les Éditions de Physique 1996 
In earlier papers $[2,8]$, using Langmuir probes, it was observed that, in the steepening process of large amplitude non-linear waves leading to ion acoustic shocks, the finite longitudinal fields can eject some of the charged particles ahead of the shock front. These particles are called "precursors" and can in turn generate ion acoustic turbulence by the usual Landau growth mechanism. The wave-front/precursor system can generate strong instabilities. In this paper, we are primarily interested in the observation of these precursor ions.

The paper is organized as follows. Section 2 briefly describes the experimental setup used to generate the electrostatic shock waves in a laboratory. Section 3 describes the LIF diagnostic modification that we have developed to obtain the time resolution necessary to study the shock propagation. Preliminary results described in Section 4 clearly show that the shock front is preceded by precursor ions which are able to destabilize it and generate bursts of turbulence. Conclusions are given in Section 5.

\section{Experimental Setup}

The experimental device is shown in Figure 1a. It is a multipolar plasma device [12] used in a double-plasma configuration [13]. It consists of two independent gas-discharge chambers operated with argon gas at a pressure of a few $10^{-4}$ Torr and separated by a plane fine grid held at floating potential. The plasma is generated by a thermoelectronic discharge with the cathode consisting of heated tungsten filaments. On the target side, the anode is constituted by the grounded walls. On the source side, the anode is made of a cylinder-plate system which can be biased with respect to ground to adjust the source plasma potential. The energy levels used by the LIF diagnostic are shown Figure 1b.

In the driver or source plasma, the electron density is equal to a few $10^{9} \mathrm{~cm}^{-3}$; in the target plasma, it is of the order of $10^{8} \mathrm{~cm}^{-3}$, as measured with plane Langmuir probes. By applying a positive or negative voltage pulse to the anode of the "driver" plasma, ions can be caused to flow into or out the "target" plasma. If the positive voltage pulse is sufficiently steep, as governed by a signal generator, a shock can develop and propagate in the target chamber. The number of shock waveforms that can be generated in this way is very large: some of them show soliton features; others show bursts of turbulence, depending on the parameters of each independent plasma. Figure 2 shows a few shock profiles. The top traces correspond to the signal applied on the cylinder-plate system. The bottom traces correspond to the signal collected on a Langmuir probe positively biased at $9 \mathrm{~V}$, and located at $20 \mathrm{~cm}$ from the entrance grid. This signal is inverted with respect to the excitation signal. In Figures $2 a$ and $2 c$ these bottom traces exhibit both:

- a direct signal synchronous with the applied signal; a small part of this signal may be due to a capacitive coupling with the probe but the main part seems to come from the ionisation process itself;

- and a retarded signal corresponding to the propagating shock.

Figure 2a shows the steepening of the shock wave and a bump in front of the shock, characteristic of the presence of precursors; Figure $2 \mathrm{~b}$ exhibits the formation of turbulence in the minimum presumably due to the presence of precursors; Figure $2 c$ corresponds to a more complicated excited signal, the steepening of the wave front is more obvious on the second oscillation; Figure $2 \mathrm{~d}$ shows a solitary structure when a strong ion beam is present. A systematic study of these phenomena is under way. 
a)

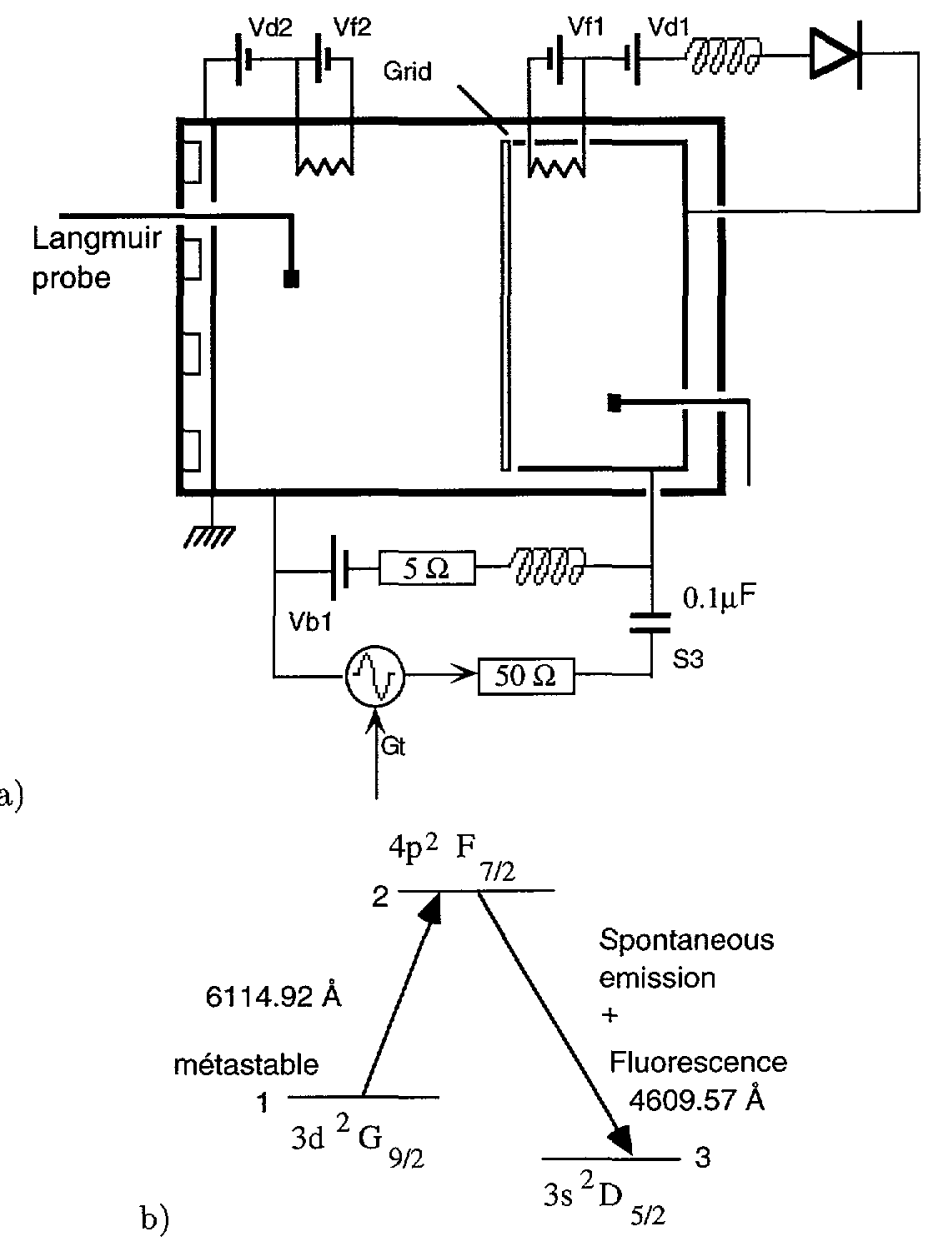

Fig. 1. - a) Sketch of the double plasma multipolar device. Vf: filament voltage; Vd: discharge voltage; Vb: cylinder-plate system bias. b) Argon energy levels diagram used by the LIF diagnostic.

\section{The Time Resolved LIF Diagnostic Setup}

The LIF diagnostic setup is shown in Figure 3. The laser induced fluorescence diagnostic [14] uses a ring dye laser pumped by a cw argon laser. This diagnostic has a spatial resolution of $6 \mathrm{~mm}^{3}$. To obtain the necessary time resolution, the lock-in amplifier previously used [14] to process the photomultiplier output has been replaced by a wide band amplifier followed by a two channels boxcar allowing to record the fluorescence signal at any time during the shock propagation. To collect the fluorescence light and discriminate the laser induced part from the light emission from the hot filaments and the spontaneous emission of the plasma, the laser output is chopped at $1.7 \mathrm{kHz}$ and the boxcar gate $\mathrm{A}$ is opened for $2 \mu \mathrm{s}$ at a given instant of the shock propagation when the laser light is present in the plasma. The other gate $(B)$ is opened at the same instant of the shock propagation but when the laser is blocked by the chopper blade. The boxcar output A-B gives a signal corresponding to the laser induced fluorescence light integrated over the gate duration. This output is processed by a dc amplifier having an integration time constant of three seconds which means averaging over approximately 5000 repetitive shocks. 
a)

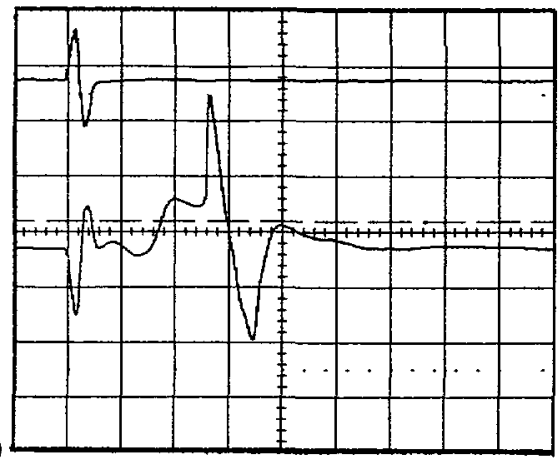

c)

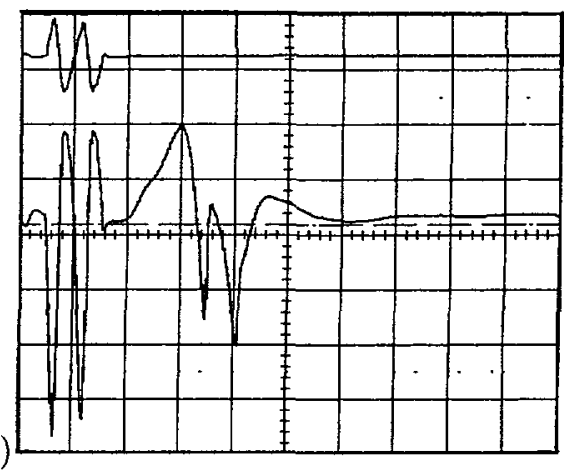

b)

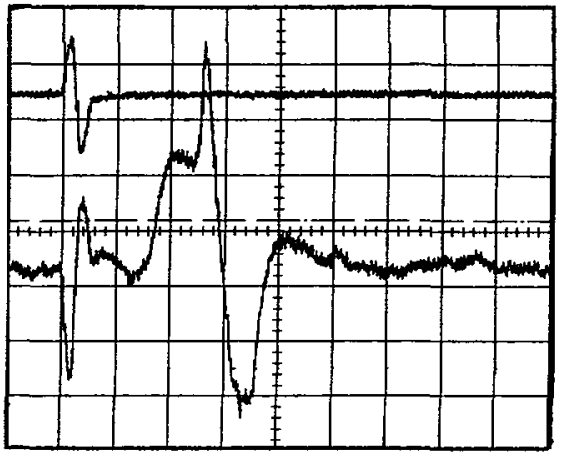

d)

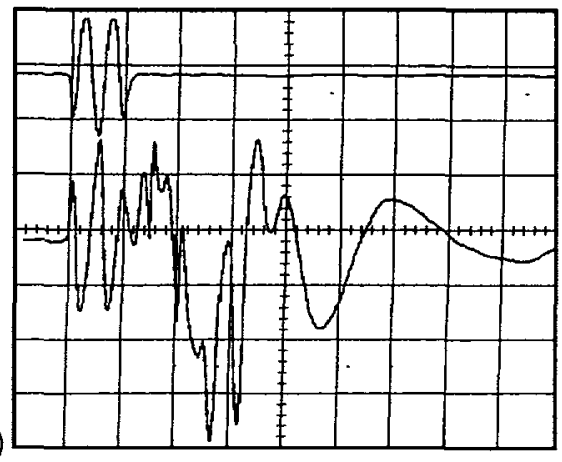

Fig. 2. - Ion acoustic shock profiles. Top trace: signal applied to the cylinder-plate system. Bottom trace: signal collected on a Langmuir probe biased at $+9 \mathrm{~V}$ located at $20 \mathrm{~cm}$ from the grid. a), b) Single pulse with different amplitudes, c), d) Triple pulse.

Figure 4 a gives a detailed time chart of the various signals that appear in Figure 3. A homemade signal box gives the triggering signals for the generator (GT) and the boxcar (BT). To avoid jitter due to the mechanical chopper used to turn on and off the laser, all the signals are synchronized with the rising edge of the chopper signal output.

To obtain accurate results, for every ion velocity class excited by the laser frequency selected by the computer, the boxcar output (A-B) corresponding to the fluorescence light is stored, with and without the shock. This is done by the micro-computer which sets the frequency output of the laser and allows or not the gate generator signal GT to be present at the signal box output (E2) for a duration which takes into account the time constant of the dc amplifier of the boxcar. In this way the influence of uncontrolled slow drift in any plasma parameters during the recording is avoided.

By controlling the $\delta t$ duration of $\mathrm{GT}$, the signal generator is able to deliver several signal waveforms as shown in Figure $4 \mathrm{~b}$.

\section{Experimental Results}

It is generally assumed $[15,16]$ that the life time of the initial argon metastable level used for the LIF diagnostic is of the order of a few microseconds. This raises the main difficulty related to the observation of the modifications of the ion velocity distribution function due to the shock propagation. Because the life time is shorter than the transit time calculated 


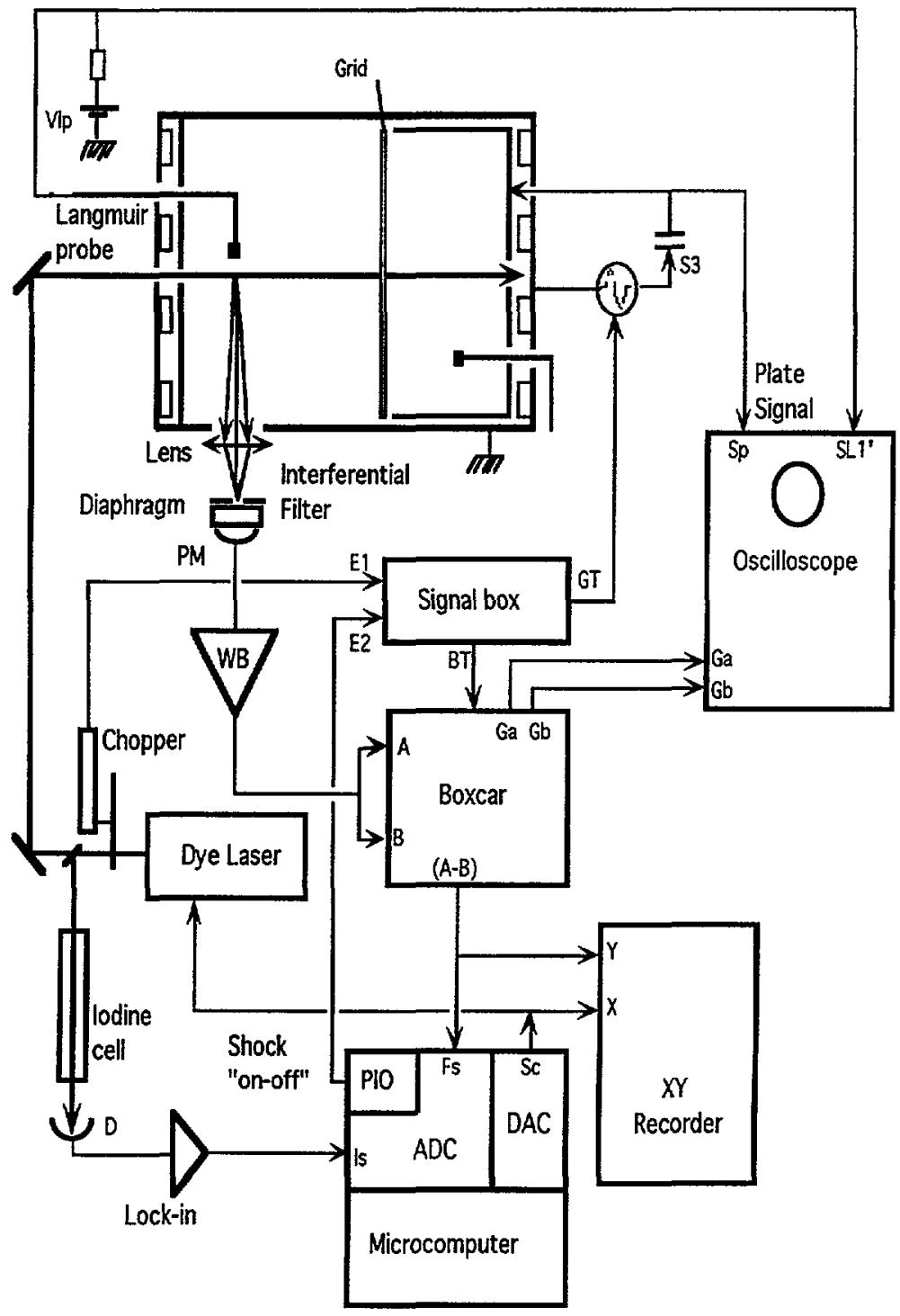

Fig. 3. - Time resolved LIF diagnostic setup.

from the velocity of the ion beam associated with the launched shock and since the probability of collisions between electrons and beam ions is very small, the fluorescent light collected at a particular position comes from ions which do not belong to the beam, and are created or excited by collisions with energetic electrons in the neighbourhood of this position. This is particularly true for the observation of precursor ions which are expected to accumulate in front of the shock.

This argument appears to be in complete contradiction with our experimental results. In fact, using the time averaged LIF diagnostic without any time resolution (a lock-in amplifier replacing the boxcar in the diagram of Fig. 3), it is very easy to observe the velocity distribution 

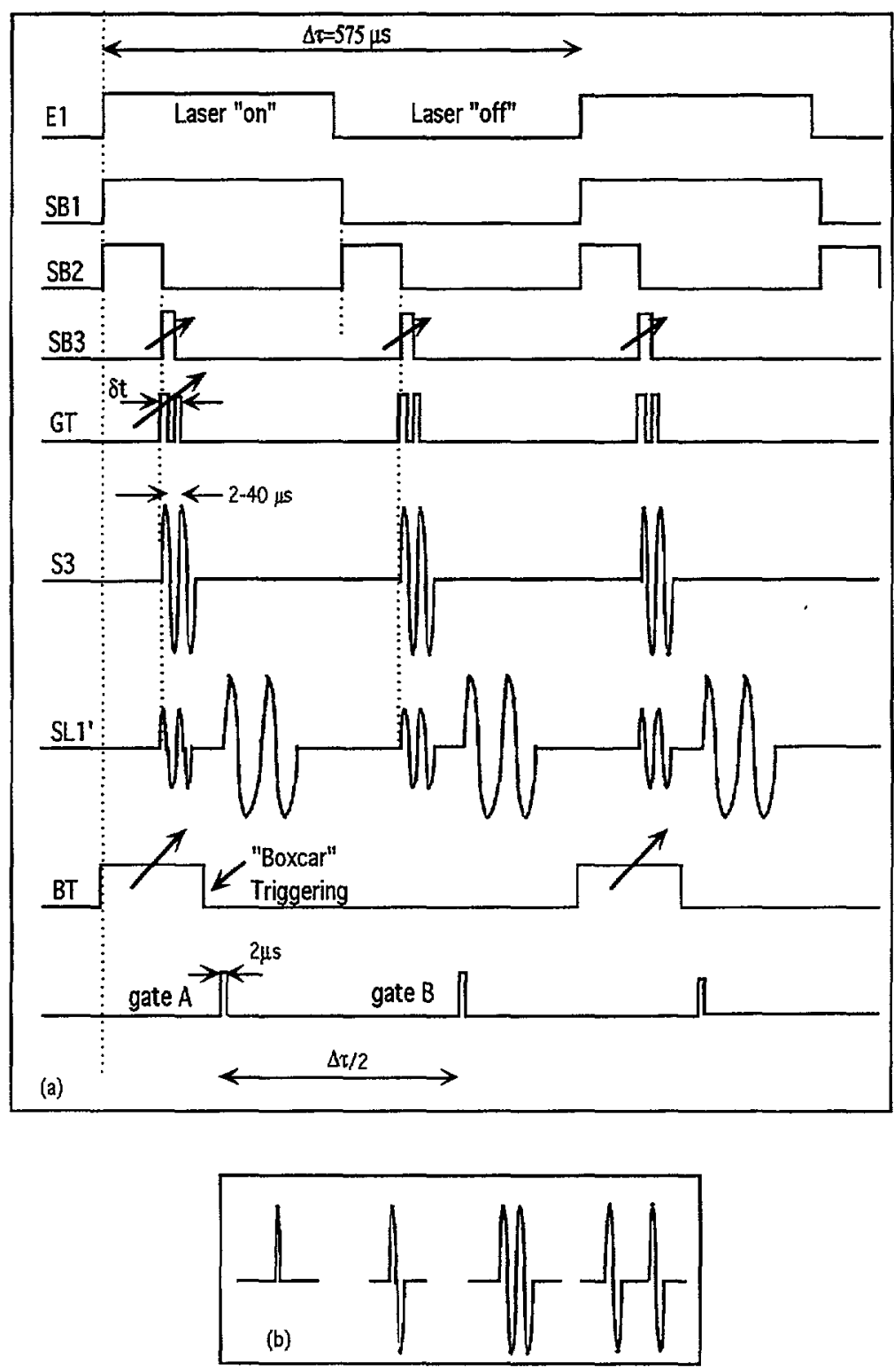

Fig. 4. - (a) Time chart of the various signals in Figure 3. (b) Typical generator output signals.

function of a permanent ion beam propagating in the target chamber [17] when a dc voltage is applied to the driver plasma. Figure 5 shows that the distribution of the ion beam can be clearly identified as well as the theoretically expected narrowing of the distribution [18] associated with the beam acceleration. So the only way to explain our observations is that the argon metastable level life time is longer than it is generally assumed.

With the time resolved LIF diagnostic described above, we can observe the modifications of the ion distribution function due to the propagation of a shock. These measurements show the 


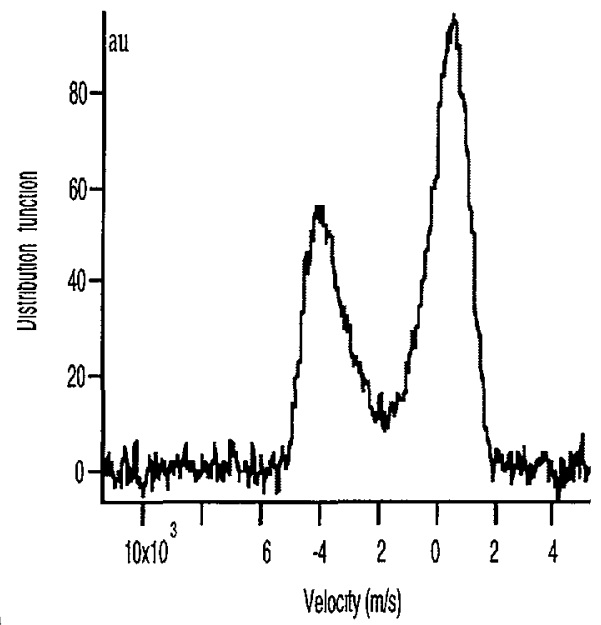

a)

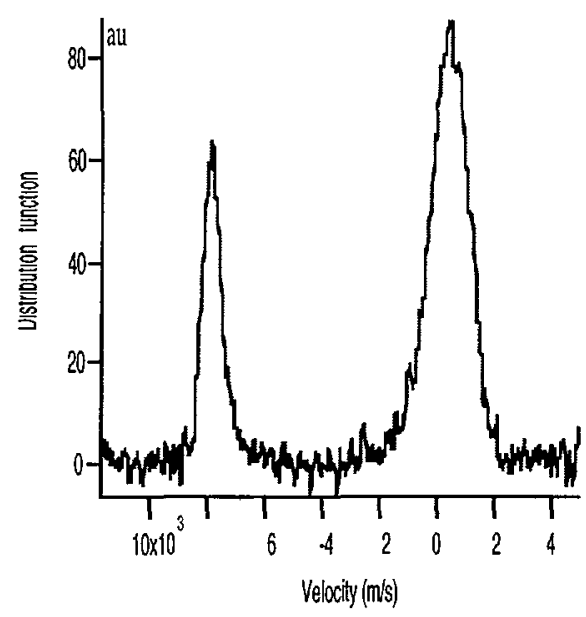

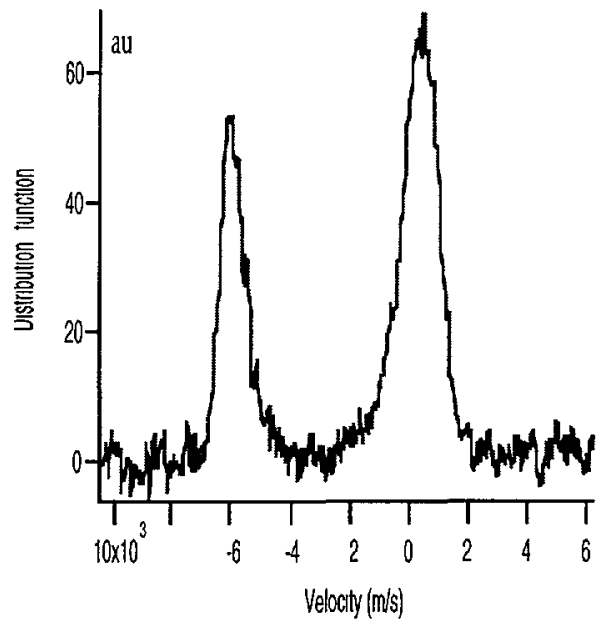

b)

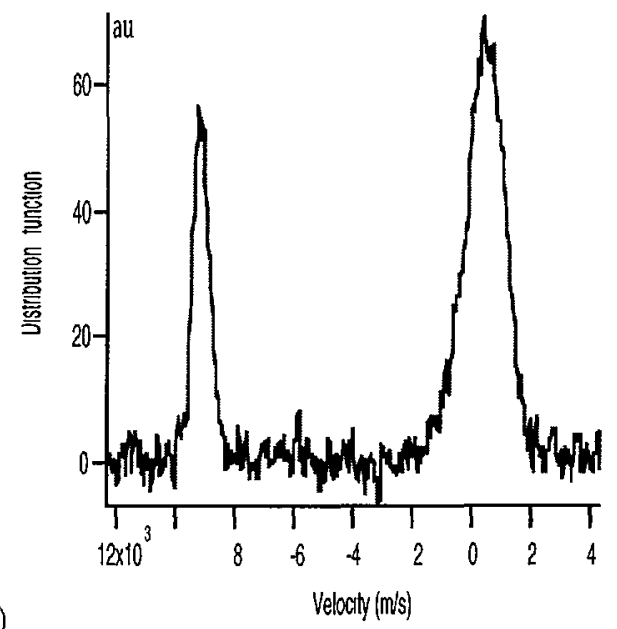

Fig. 5. - Ion velocity distribution functions measured at $5 \mathrm{~cm}$ from the entrance grid with an ion beam injected into the target chamber. a) $\mathrm{Vb}=5 \mathrm{~V}, \mathrm{~b}) 10 \mathrm{~V}, \mathrm{c}) 15 \mathrm{~V}$ and d) $20 \mathrm{~V}$.

presence of trapped ions behind the front shock and also precursor ions when an electrostatic shock propagates in the target plasma.

Figure 6 shows the fluorescence light recorded in the target chamber at $20 \mathrm{~cm}$ from the separation grid, when the gates of the boxcar are set in the precursor region, in front of the shock. We can see that the main distribution function is preceded by a small bump the mean velocity of which is approximately equal to twice the ion acoustic speed. This is the expected velocity range for the precursor ions [19].

It is important to point out the method used to obtain the two curves of Figure 6 which give the ion velocity distribution function i.e. the collected fluorescent light as a function of the laser frequency, when the shock is alternatively turned on and off: to each point of the upper curve obtained when the shock is on, corresponds a point of the lower curve obtained by switching off the shock for the same laser frequency. Of course, as said before, at each switching, we 


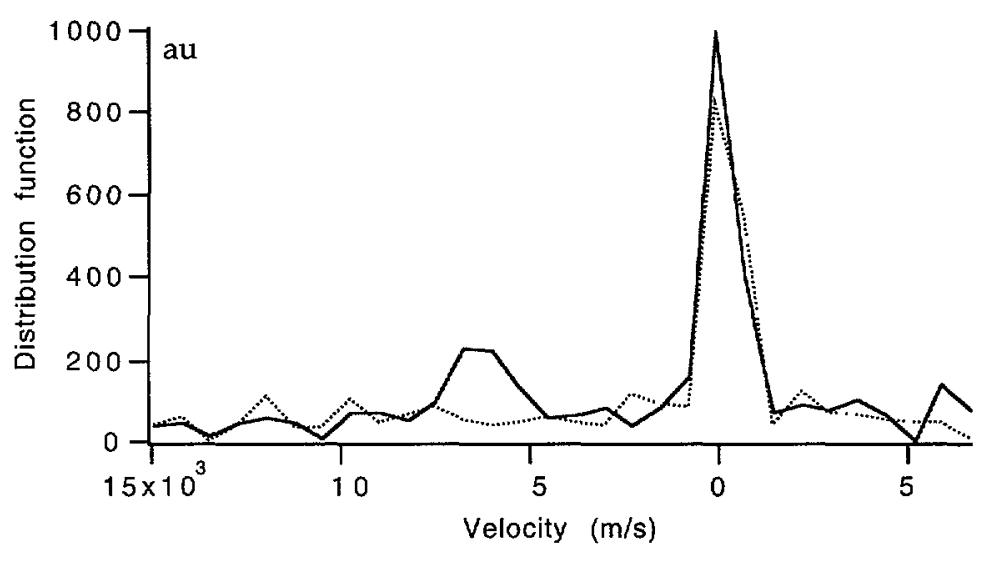

Fig. 6. - Ion velocity distribution function with precursor ions measured in front of the shock, at $20 \mathrm{~cm}$ from the entrance grid. Dashed (resp. continuous) line: without (resp. with) ion acoustic shock.

have taken into account the recovery time constant of the boxcar. Therefore we are sure that the observed little bump is due to the shock and does not result from an uncontrolled slow modification of the plasma parameters during the measurement time.

Concerning the ability to reproduce these data, it appears that the conditions in which the precursors are observed are very sharp, a small change in one of the parameters of the two plasmas can totally change the conditions of observation. The other point is that the boxcar gates are adjusted on the Langmuir probe signal: a small misalignment between the probe and the line of sight of the fluorescence detection block can induce shift between the position of the gate and the observed phenomenon. This problem can be avoided by using a multichannel scaler in place of the boxcar. This is a technique that we will further develop.

\section{Conclusion}

With a laser induced fluorescence diagnostic having both space and time resolution, we have been able to observe the velocity distribution function of the precursor ions of an electrostatic collisionless ion acoustic shock. This result shows that a systematic experimental study of non-linear processes associated with the propagation of large amplitude ion acoustic waves is possible. It will be the subject of forthcoming publications.

\section{Acknowledgments}

The authors are indebted to B. Squizzaro and A. Totin for their skillful technical assistance. They acknowledge stimulating discussions with J. Derouard and N. Sadeghi. One of the authors (R.A. S.) was supported by the Université de Provence during his stay in Marseille. 


\section{References}

[1] Taylor R.J., Baker D.R. and Ikezi H., Phys. Rev. Lett. 24 (1970) 206.

[2] Wong A.Y. and Means R.W., Phys. Rev. Lett. 27 (1971) 973.

[3] Roberts K.V. and Berk H.L., Phys. Rev. Lett. 19 (1967) 297.

[4] Kako M., Taniuti T. and Watanabe T., J. Phys. Soc. Jpn 31 (1971) 1820.

[5] Sakanaka P.H., Phys. Fluids 15 (1972) 1323.

[6] Bonhomme G., Pierre T., Leclert G. and Trulsen J., Plasma Phys. Contr. Fusion 33 (1991) 507.

[7] Judice C.N., Decker J.F. and Stern R.A., Phys, Rev. Lett. 30 (1973) 267.

[8] Stern R.A. and Decker J.F., Phys. Rev. Lett. 27 (1971) 1266.

[9] Jolly J., J. Phys. III France 5 (1995) 1089.

[10] Stern R.A. and Johnson J.A., Phys. Rev. Lett. 34 (1975) 1548.

[11] Hill D.N., Fornaca S. and Wickam M.G., Rev. Sci. Instrum. 54 (1983) 309.

[12] Limpaecher R. and MacKenzie K.R., Rev. Sci. Instrum. 44 (1973) 726.

[13] Ikezi H. and Taylor R.J., Phys. Rev. Lett. 22 (1969) 923.

[14] Bachet G., Chérigier L., Carrère M. and Doveil F., Phys. Fluids B5 (1993) 3097.

[15] Chatain F., Ph D Thesis, Université Joseph Fourier de Grenoble, France (1995).

[16] Andereg F., Ph D Thesis, Université de Lausanne, Suisse (1988).

[17] Spinelli F., Internal report, Université Paul Sabatier de Toulouse, France (1993).

[18] Wong A.Y., Experimental Plasma Physics Course, University of California Los Angeles (1977).

[19] Tidman D.A. and Krall N.A., Shock Waves in Collisionless Plasmas (Wiley-Interscience, New York, 1971). 